\title{
STABILITY FOR DISSIPATIVE MAGNETO-ELASTIC SYSTEMS
}

\author{
REINHARD RACKE \\ Department of Mathematics, University of Konstanz \\ 78457 Konstanz, Germany \\ E-mail: reinhard.racke@uni-konstanz.de
}

\begin{abstract}
In this survey we first recall results on the asymptotic behavior of solutions in classical thermoelasticity. Then we report on recent results in linear magneto-thermo-elasticity and magneto-elasticity, respectively.

1. Introduction. The time-dependent system of elasticity is known to be of hyperbolic type, while that of heat conduction is parabolic. As third system we consider Maxwell's equations where displacement currents are neglected, thus resulting in a parabolic system for the magnetic field.

We are interested in the asymptotic behavior of solutions as time tends to infinity for different coupling of the systems as there are: thermo-elasticity, magneto-elasticity and magneto-thermo-elasticity. Due to the coupling of different types of differential equations with in part strongly different kind of behavior for example in bounded domains, the interesting question arises: what is the predominating part, and how is this expressed in terms of decay rates of the solutions?

We start with recalling well-known results in thermo-elasticity, then we report on recent results obtained in magneto-thermo-elasticity and in magneto-elasticity, respectively.
\end{abstract}

\section{Typical features of thermo-elasticity. Let}

$$
u=u(t, x) \in \mathbb{R}^{n}, n=1,2,3, \quad \theta(t, x) \in \mathbb{R},
$$

$t \geq 0, x \in \Omega \subset \mathbb{R}^{n}$, describe the displacement vector and the temperature difference, respectively, in a homogeneous, isotropic, linear medium. Then

$$
\begin{aligned}
u_{t t}-\mu \Delta u-(\lambda+\mu) \nabla \operatorname{div} u+\gamma \nabla \theta=0 & {\left[F\left(\nabla^{2} u, \ldots\right)\right], } \\
\theta_{t}-\kappa \Delta \theta+\gamma \operatorname{div} u_{t}=0 & {\left[f\left(\nabla u_{t}, \nabla^{2} \theta^{2}, \ldots\right)\right] }
\end{aligned}
$$

2000 Mathematics Subject Classification: 74F05, 74F15.

The paper is in final form and no version of it will be published elsewhere. 
plus initial and boundary conditions, e.g.

$$
u=0, \quad \theta=0 \text { on } \partial \Omega
$$

([...] indicates a possible nonlinear structure).

Typical results. Let

$$
E(t):=\frac{1}{2} \int_{\Omega}\left(\left|u_{t}\right|^{2}+\mu|\nabla u|^{2}+(\lambda+\mu)|\operatorname{div} u|^{2}+|\theta|^{2}\right)(t, x) d x
$$

denote the energy at time $t$. Then we have:

$n=1$ :

- $\Omega=(0,1): E(t) \leq d_{1} e^{-d_{2} t} E(0)$, with positive constants $d_{1}, d_{2}$.

Dissipation through heat conduction leads to overall exponential decay.

- $\Omega=\mathbb{R}:\left\|u_{t}(t), \theta(t)\right\|_{L^{\infty}} \leq C \cdot(1+t)^{-1 / 2}, C>0$ constant.

Behavior like heat conduction.

$n=2,3$ :

- Reflecting rays imply slow decay: Whenever there exist reflecting rays that is a two-periodic orbit of the billiard in the domain, there is no uniform decay of the energy.

- $\Omega=B(0,1)$ (unit ball), radial symmetry of the data: Energy decays exponentially.

- $E(t) \rightarrow 0 \Leftrightarrow\left(\left\{\begin{aligned} \Delta u+\lambda u=0 \\ u_{\mid \partial \Omega}=0 \\ \operatorname{div} u=0\end{aligned}\right\} \Rightarrow u=0\right)=:(*)$

(*) holds if $\Delta v+\lambda v=0, v_{\mid \partial \Omega}=0$ (scalar) has only simple eigenvalues.

- $\Omega=\mathbb{R}^{3}: \quad U=U^{P}+U^{S} \in \overline{\nabla H^{1}} \oplus D_{0}$, where $D_{0}$ denotes divergence-free vector fields.

$$
\begin{aligned}
& U_{t t}^{S}-\mu \Delta U^{S}=0: \text { no dissipation. } \\
& \left(U^{S}, \theta\right): \text { like heat equation. }
\end{aligned}
$$

We remark that nonlinear systems in $\mathbb{R}^{1}$ are globally well-posed for small data, in $\mathbb{R}^{3}$ under radial symmetry in bounded domains (for small data), for $\Omega=\mathbb{R}^{3}$ if certain nonlinearities are excluded.

The results recalled in this section have been obtained over the last 30 years, among the contributors are Dafermos, Henry, Jiang, Kawashima, Kim, Koch, Lebeau, Lopes, Muñoz Rivera, Perissinitto, Shen, Shibata, Slemrod, Zheng, Zuazua and the author. A comprehensive survey with detailed references can be found in [4].

3. Magneto-thermo-elasticity. We consider the system of magneto-thermo-elasticity for the displacement vector $u$, the temperature difference $\theta$ and the magnetic field $h$ :

$$
\begin{gathered}
u_{t t}-E u-\alpha[\nabla \times h] \times \vec{H}+\gamma \nabla \theta=0, \\
h_{t}-\Delta h-\beta \nabla \times\left[u_{t} \times \vec{H}\right]=0, \\
\theta_{t}-\kappa \Delta \theta+\gamma \operatorname{div} u_{t}=0, \\
E u=\left[\left(C_{i j k l} u_{, l}^{k}\right)_{, j}\right]_{i=1,2,3},
\end{gathered}
$$


in the homogeneous isotropic case under consideration we have

$$
E u=\mu \Delta u+(\lambda+\mu) \nabla \operatorname{div} u
$$

and $\vec{H}=(0,0, H)^{\prime}$. Additionally we have the initial conditions

$$
u(0, x)=u_{0}(x), u_{t}(0, x)=u_{1}(x), h(0, x)=h_{0}(x), \theta(0, x)=\theta_{0}(x) .
$$

First we shall consider the Cauchy problem, then the bounded domain case for which we shall assume the following setup.

$\Omega \subset \mathbb{R}^{3}$ with smooth boundary $\Gamma=\partial \Omega$ and $\Gamma=\Gamma_{0} \cup \Gamma_{1}$ with $\Gamma_{0} \cap \Gamma_{1} \neq \emptyset$. Assume that there is $x_{0} \in \Omega$ such that

$$
\begin{aligned}
& \Gamma_{0}=\left\{x \in \Gamma:\left(x-x_{0}\right) \cdot \nu(x) \leq 0\right\}, \\
& \Gamma_{1}=\left\{x \in \Gamma:\left(x-x_{0}\right) \cdot \nu(x) \geq a>0\right\} .
\end{aligned}
$$

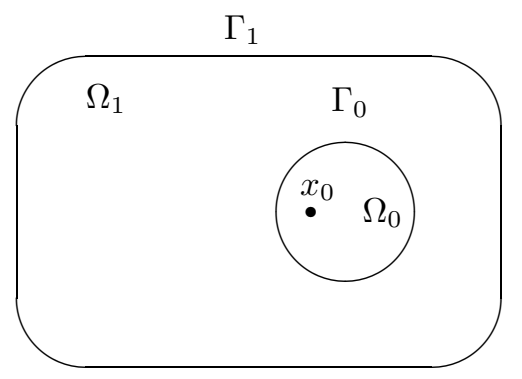

$$
\Omega=\Omega_{1} \backslash \Omega_{0}
$$

Boundary conditions for $h$ and $\theta$ :

$$
\nu \times(\nabla \times h)=0, \nu \cdot h=0, \theta=0 \quad \text { on } \Gamma
$$

and a memory type boundary condition for $u$ :

$$
\begin{gathered}
u=0 \quad \text { on } \Gamma_{0}, \quad u+r * \partial_{\nu} u=0 \quad \text { on } \Gamma_{1} . \\
\partial_{\nu} u:=\left(C_{i j k l} u_{, l}^{k} \nu^{j}\right)_{i=1,2,3}-\alpha H h^{3} \nu+\alpha H \nu^{3} h, \\
(r * f)(t):=\int_{0}^{t} r(t-s) f(s) d s \\
\partial_{\nu} u=-\tau u_{t}-\tau g * u_{t}, \quad \tau>0 .
\end{gathered}
$$

First we shall assume that $g$ essentially decays exponentially, i.e. for $t \geq 0$

$$
\left.\begin{array}{rl}
0 & <g(t) \leq c_{0} e^{-g_{0} t}, \\
-c_{1} g(t) & \leq g^{\prime}(t) \leq-c_{2} g(t), \\
-c_{3} g^{\prime}(t) & \leq g^{\prime \prime}(t) \leq-c_{4} g^{\prime}(t)
\end{array}\right\}
$$

with positive constants $c_{j}$. Example:

$$
\tilde{g}(t)=c_{0} e^{-g_{0} t} .
$$


Second, we shall consider polynomially decaying kernels satisfying for $t \geq 0$

$$
\left.\begin{array}{rl}
0 & <g(t) \leq b_{0}(1+t)^{-p}, \\
-b_{1} g(t)^{(p+1) / p} & \leq g^{\prime}(t) \leq-b_{2} g(t)^{(p+1) / p}, \\
-b_{3}\left|g^{\prime}(t)\right|^{(p+2) /(p+1)} & \leq g^{\prime \prime}(t) \leq-b_{4}\left|g^{\prime}(t)\right|^{(p+2) /(p+1)}
\end{array}\right\}
$$

with positive constants $b_{j}$. Example:

$$
\bar{g}(t)=b_{0}(1+t)^{-p} .
$$

3.1. The Cauchy problem

$$
\begin{gathered}
u_{t t}-\mu \Delta u-(\mu+\lambda) \nabla \operatorname{div} u+\alpha H \nabla h^{3}-\alpha H \frac{\partial h}{\partial x_{3}}+\gamma \nabla \theta=0, \\
h_{t}-\Delta h-\beta H \frac{\partial u_{t}}{\partial x_{3}}+\beta H\left(0,0, \operatorname{div} u_{t}\right)^{\prime}=0, \\
\theta_{t}-\kappa \Delta \theta+\gamma \operatorname{div} u_{t}=0 .
\end{gathered}
$$

Using Fourier transform

$$
v:=\hat{u}, \quad w:=\hat{h}, \quad \psi:=\hat{\theta} .
$$

we get

$$
\begin{gathered}
v_{t t}+\mu|\eta|^{2} v+(\mu+\lambda)(\eta v) \eta-i \alpha H \eta w^{3}+i \alpha H \eta_{3} w-i \gamma \psi \eta=0 \\
w_{t}+|\eta|^{2} w+i \beta H \eta_{3} v_{t}-\beta H\left(0,0, v_{t} \eta\right)^{\prime}=0 \\
\psi_{t}+\kappa|\eta|^{2} \psi-i \gamma v_{t} \eta=0
\end{gathered}
$$

Next,

$$
\frac{d}{d t} \mathcal{E}_{1}(t)=-\frac{\alpha}{\beta}|\eta|^{2}|w|^{2}-\kappa|\eta|^{2}|\psi|^{2}
$$

where

$$
\mathcal{E}_{1}(t):=\frac{1}{2}\left\{\left|v_{t}\right|^{2}+\mu|\eta|^{2}|v|^{2}+(\mu+\lambda)(\eta v)^{2}+\frac{\alpha}{\beta}|w|^{2}+|\psi|^{2}\right\}(t) .
$$

Let

$$
\mathcal{A} \equiv \mathcal{A}(\eta):=|\eta|^{2}\left(\frac{1}{\eta_{1}^{2}}+\frac{1}{\eta_{3}^{2}}\right) .
$$

THEOREM 1. If the initial data satisfy

$$
\int_{\mathbb{R}^{n}}\left[\frac{\left(1+|\eta|^{2}\right) \mathcal{A}^{2}}{|\eta|^{2}}\right]^{m+1} \mathcal{E}_{1}(0, \eta) d \eta<\infty
$$

$m \in \mathbb{N}_{0}$, then the energy term

$$
E(t):=\frac{1}{2} \int_{\mathbb{R}^{n}}\left\{\left|u_{t}\right|^{2}+\mu|\nabla u|^{2}+(\mu+\lambda)|\operatorname{div} u|^{2}+\frac{\alpha}{\beta}|h|^{2}+|\theta|^{2}\right\}(t, x) d x
$$

associated to a solution $(u, h, \theta)$ to the Cauchy problem decays polynomially:

$$
E(t)=\mathcal{O}\left(t^{-m}\right) \quad \text { as } t \rightarrow \infty .
$$


3.2. The bounded domain case. Assuming div $h_{0}=0$, we have

$$
\operatorname{div} h(t, \cdot)=0 \quad \text { for all } t \geq 0 \text {. }
$$

Let

$$
\begin{aligned}
F(t):=\frac{1}{2} \int_{\Omega}\left(\left|u_{t}\right|^{2}+C_{i j k l} u_{, l}^{k} u_{, j}^{i}\right. & \left.+\frac{\alpha}{\beta}|h|^{2}+|\theta|^{2}\right)(t, x) d x \\
& -\frac{\tau}{2} \int_{\Gamma_{1}}\left(g^{\prime} \square u\right)(t, z) d z+\frac{\tau}{2} g(t) \int_{\Gamma_{1}}|u|^{2}(t, z) d z,
\end{aligned}
$$

where

$$
(f \square \varphi)(t):=\int_{0}^{t} f(t-s)|\varphi(t)-\varphi(s)|^{2} d s .
$$

THEOREM 2. Let $g$ be an exponentially decaying resolvent kernel. Then the energy $F$ decays exponentially, i.e.

$$
\exists d_{0}, d_{1}>0 \quad \forall t \geq 0 \quad F(t) \leq d_{0} e^{-d_{1} t} F(0) .
$$

TheOREM 3. Let $g$ be a polynomially decaying resolvent kernel. Then the energy $F$ decays polynomially, i.e.

$$
\exists d_{2}>0 \quad \forall t \geq 0 \quad F(t) \leq \frac{d_{2}}{(1+t)^{p}} F(0)
$$

The results in this section have been obtained in collaboration with J. E. Muñoz Rivera [5]. For previous or related work see $[1,2,3,7,8,9]$ and further references in [5].

4. Magneto-elasticity. Let $\Omega \subset \mathbb{R}^{2}$ be bounded. The system of magneto-elasticity for the displacement $u=\left(u^{1}, u^{2}, 0\right)=u\left(t, x_{1}, x_{2}\right)$ and the magnetic field $h=\left(h^{1}, h^{2}, 0\right)$ to be considered is the following (here $\left.\vec{H}=(H, 0,0)^{\prime}\right)$ :

$$
\begin{gathered}
u_{t t}-\mu \Delta u-(\lambda+\mu) \nabla \operatorname{div} u-\alpha(\nabla \times h) \times \vec{H}=0, \\
h_{t}-\Delta h-\beta \nabla \times\left(u_{t} \times \vec{H}\right)=0, \\
u(t=0)=u_{0}, u_{t}(t=0)=u_{1}, h(t=0)=h_{1}, \\
u=0, \nu \cdot h=0, \nu \times(\nabla \times h)=0 \quad \text { on } \partial \Omega .
\end{gathered}
$$

Here, $\nu$ denotes again the exterior normal and we have $\operatorname{div} h=0$. Admissible domains are those that are homeomorphic to the unit ball and for which one of the following three conditions I-III is satisfied:

I. $\Omega$ is the union of finitely many rectangles with axes parallel to the $x_{1}$ - and $x_{2}$-axes, respectively, see Figure 1.

II. $\Omega$ satisfies $\nu_{1} \nu_{2}=0$ in the first quadrant (where $x_{1} \geq 0$ and $x_{2} \geq 0$ ) and in the third quadrant (where $x_{1} \leq 0$ and $x_{2} \leq 0$ ). In the second and fourth quadrants $\Omega$ satisfies $x \nu \geq \delta_{0}>0$, for some $\delta_{0}$, see Figure 2 .

III. $\Omega$ satisfies $\nu_{1} \nu_{2}=0$ in the second and fourth quadrants. In the first and third quadrants $\Omega$ satisfies $x \nu \geq \delta_{0}>0$, for some $\delta_{0}$, see Figure 3 . 


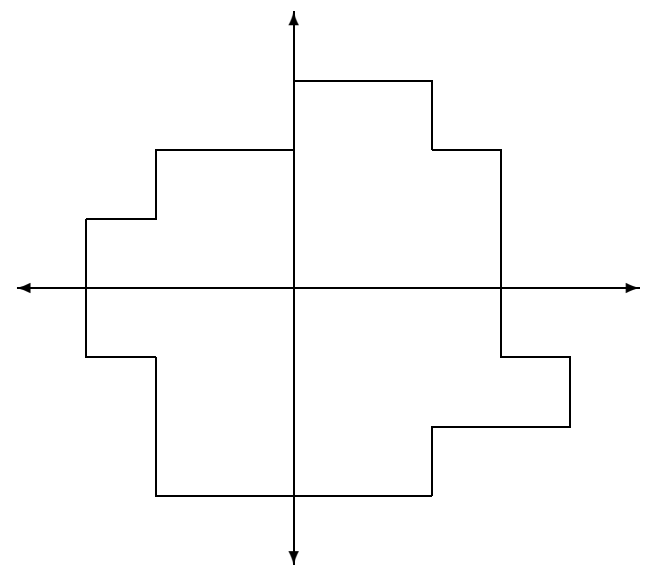

Figure 1. Type I

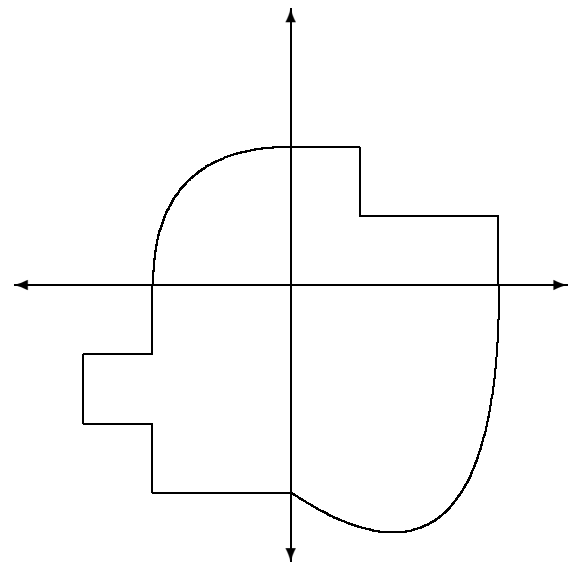

Figure 2. Type II

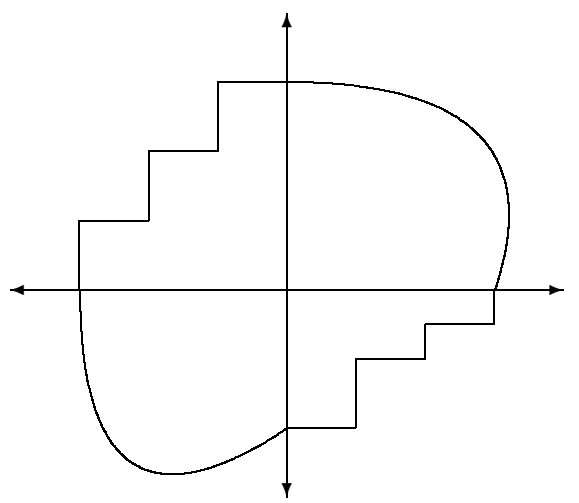

Figure 3. Type III

Let

$$
\begin{gathered}
E(t) \equiv E(t ; u, h):=\frac{1}{2} \int_{\Omega}\left|u_{t}\right|^{2}+\mu|\nabla u|^{2}+(\mu+\lambda)|\operatorname{div} u|^{2}+\frac{\alpha}{\beta}|h|^{2} d x, \\
E_{j}(t):=E\left(t ; \partial_{t}^{j} u, \partial_{t}^{j} h\right), j \geq 0 .
\end{gathered}
$$

Then a polynomial decay of the energy is described by

THEOREM 4. There exists $d>0$ such that for all $t \geq 0$ we have $E(t) \leq d \sum_{j=0}^{7} E_{j}(0) \cdot \frac{1}{t}$.

(Use $\omega:=\nabla \times h=\partial_{1} h^{2}-\partial_{2} h^{1}, \omega_{\mid \partial \Omega}=0$.)

The results in this section have been obtained in collaboration with J. E. Muñoz Rivera [6]. For previous or related work see $[1,7]$ and further references in [6]. 


\section{References}

[1] E. Andreou and G. Dassios, Dissipation of energy for magnetoelastic waves in a conductive medium, Quart. Appl. Math. 55 (1997), 23-39.

[2] M. Ciarletta, A differential problem for heat equation with a boundary condition with memory, Appl. Math. Lett. 10 (1997), 95-101.

[3] M. Fabrizio and A. Morro, A boundary condition with memory in electromagnetism, Arch. Rational Mech. Anal. 136 (1996), 359-381.

[4] S. JIANG and R. RACKE, Evolution Equations in Thermoelasticity, Chapman Hall/CRC Monogr. Surv. Pure Appl. Math. 112, Chapman \& Hall/CRC, Boca Raton, 2000.

[5] J. E. MuÑoz Rivera and R. RACKe, Magneto-thermo-elasticity - large time behavior for linear systems, Adv. Differential Equations 6 (2001), 359-384.

[6] J. E. Muñoz Rivera and R. RaCke, Polynomial stability in two-dimensional magnetoelasticity, IMA J. Appl. Math. 66 (2001), 269-283.

[7] G. Perla Menzala and E. Zuazua, Energy decay of magnetoelastic waves in a bounded conductive medium, Asymptot. Anal. 18 (1998), 349-362.

[8] T. QIN, Global solvability of nonlinear wave equation with a viscoelastic boundary condition, Chinese Ann. Math. Ser. B 14 (1993), 335-346.

[9] T. QIN, Breakdown of solutions to nonlinear wave equation with a viscoelastic boundary condition, Arabian J. Sci. Engrg. 19 (1994), 195-202. 